\title{
Políticas públicas en educación a distancia y el logro \\ de competencias en estudiantes de educación superior del IESTP \\ "Manuel Arévalo Cáceres" -los olivos, Lima, Perú
}

\author{
Enrique Cárdenas Díaz \\ ccardenasdi68@ucvvirtual.edu.pe \\ Doctor en Gestión Pública y Gobernabilidad \\ en la Universidad César Vallejo \\ Lima - Perú
}

\section{RESUMEN}

OBJETIVO: Estudiar la incidencia de la Educación a Distancia como Política Pública en el Logro de Competencias en estudiantes de Educación Superior. METODOLOGÍA: Bajo un estudio cuantitativo básico aplicado de nivel correlacional, y un diseño no experimental transeccional descriptiva, la técnica empleada fue la encuesta como instrumento de recolección de datos el cuestionario. Asimismo, la población en estudio es de 115 estudiantes del último año de estudios del Área Académica de Computación e Informática y docentes, del IESTP. "Manuel Arévalo Cáceres" -Los Olivos, Lima, Perú. CONCLUSIÓN Y RESULTADOS: El objetivo general, las políticas públicas en la educación a distancia se relacionan con el logro de competencias en los sujetos de estudio, es aceptada con un nivel de significancia de 0,000 y un Chi cuadrado de Pearson de 15,369. En colaboración de la correlación de Rho de Spearman, se obtuvo la cifra de 0.413 , cuyo valor es de nivel moderado, por lo tanto, se valida el nexo entre las variables.

Palabras clave: políticas públicas; educación a distancia y competencias. 


\title{
Public policy in distance education and the achievement of competencies in higher education students IESTP "Manuel Arévalo Cáceres" -los olivos, Lima, Perú
}

\begin{abstract}
OBJECTIVE: To study the incidence of Distance Education as a Public Policy in the Achievement of Competences in Higher Education students. METHODOLOGY: Under a basic quantitative study applied at a correlational level, and a descriptive transectional non-experimental design, the technique used was the survey and the data collection instrument was the questionnaire. Likewise, the population under study is 115 students of the last year of studies of the Academic Area of Computing and Informatics and teachers of the IESTP. "Manuel Arévalo Cáceres" -Los Olivos, Lima, Peru. CONCLUSION AND RESULTS: The general objective, public policies in distance education are related to the achievement of competencies in the study subjects, it is accepted with a significance level of 0.000 and a Pearson's Chi-square of 15.369. In collaboration with the Spearman Rho correlation, the figure of 0.413 was obtained, whose value is of a moderate level, therefore, the nexus between the variables is validated.
\end{abstract}

Keywords: Public Policy, Distance Education and Competencies.

Artículo recibido: 02 noviembre. 2021 Aceptado para publicación: 28 noviembre 2021 Correspondencia: ccardenasdi68@ucvvirtual.edu.pe Conflictos de Interés: Ninguna que declarar 
Cárdenas Díaz...

\section{INTRODUCCIÓN}

El mundo se ha visto sumergido en incertidumbre y temor por la llegada de un virus que ha obligado a realizar cambios importantes en las conductas de la sociedad, la COVID19, estos cambios han permitido que la sociedad pueda reordenarse y restructurarse, sin embargo, también ha ocasionado algunos problemas para los cuales los países no estaban listos de afrontar y solucionar (Dauzón \& Izquierdo, 2020). Muchos sectores se han visto obligados a cambiar y atraer nuevos métodos para la realización de sus actividades, uno de ellos ha sido la industria educativa, debido a que este ha tenido que mutar al aprendizaje y las clases al ámbito virtual en vista de que las escuelas cerraron y muchas de ellas no tenían los recursos para seguir dictando clases de manera segura y eficiente (Avalos \& Brenes, 2021).

Karasel et al. (2020) hace referencia a que el uso de las Tecnologías de la Información y las Comunicaciones (TIC) se ha convertido en una herramienta para que los profesores y alumnos mantengan una comunicación remota, si bien era imprescindible que el docente orientara a sus estudiantes de manera directa y presencial, con las nuevas tecnologías surge una nueva oportunidad. Sin embargo, muchos de los implicados no se encontraban listos debido a una desconexión pedagógica, ya que el paso a la virtualidad necesitaba de un personal formado y capacitado para el dictado, pese a que muchas escuelas habían implementado ya esta enseñanza como un acompañamiento (Sierralta, 2021).

Además, según estudios las TIC son herramientas que permiten que el aprendizaje mejore, sin embargo, otros autores dictaminan que mezclar la enseñanza con tecnología resulta contraproducente porque produce en los alumnos cansancio visual, mala postura, dependencia, distracción, entre otras desventajas (Higuera \& Rivera, 2021). Sin embargo, este paso a la virtualidad de las clases se ha dado en países en desarrollo de manera gradual, pero con la llegada de la COVID-19 esto se tuvo que adecuar rápidamente para que los alumnos pudieran seguir aprendiendo, ya que permitía que los estudiantes desarrollen cierta autonomía, inmediatez de comunicación, diversidad de recursos de aprendizaje, trabajo didáctico y colaborativo, etc (Chiappe \& Wills, 2021).

Boboc y Koc (2019) indican que la educación a distancia ha evolucionado y se basa en los siguientes supuestos: atender a las personas que no son prioridad en los entornos tradicionales, debe ser una herramienta estratégica, formación continua al personal para una enseñanza didáctica, entender que existe una diversidad de alumnos y necesidades, 
entre otros. Además, ofrece una comunicación que puede darse desde cualquier lugar y en cualquier momento, sin embargo, los recursos virtuales en la educación son variados, desde elementos sincrónicos y asíncrono, ambos permiten que los estudiantes puedan aprender de manera flexible. Por otro lado, si bien ofrece diferentes alternativas sigue siendo un reto, ya que limita el contacto social que es tan importante, se convierte en un aprendizaje incompleto, educación no formal y el déficit en el uso de herramientas, esto puede traer problemas en la enseñanza, agravando los fatores que ya se tenían (Sood, 2016).

En los últimos años se ha potenciado el sector educativo, sin embargo, aún no se ha logrado reducir la brecha del acceso al servicio, lo cual ha desencadenado una falta de calidad entre el sector público y privado, asimismo, los recursos tecnológicos no se encuentran disponibles para la educación virtual, ya que antes de este cambio digital las personas no tenían las capacidades de obtener estas herramientas porque no era algo de prioridad (Gómez \& Escobar, 2021). Pese a que existe la Resolución Viceministerial $\mathrm{N}^{\circ}$ 084-2020 publicada el 31 de marzo del año 2020, la cual hace referencia a que el Gobierno actual debe priorizar y regular las políticas en educación, así como dictar e implementar estrategias de calidad y herramientas para cumplir las exigencias necesarias en el sector. Sin embargo, con la llegada de la pandemia se estableció el Decreto Supremo $N^{\circ} 008$ 2020-SA, el cual declara la emergencia sanitaria pero los entes públicos son derivados como los encargados de asistir al sistema educativo para el cumplimiento de sus actividades, en acompañamiento con el Decreto de Urgencia $\mathrm{N}^{\circ}$ 026-2020, precisando que los centros públicos y privados deben ofrecer una educación virtual remota.

Pires et al. (2020) precisa que la situación de la pandemia de COVID-19 es grave, ya que este cambio necesita de un equipo capacitado para detectar las capacidades de los alumnos y los medios necesarios, donde todos puedan acceder a un internet estable, incluso la grabación de las clases puede resultar beneficioso, pero también evita una asistencia a clases continua o una falta de interacción que garantice un aprendizaje eficaz. Por tal motivo, resulta importante que el Estado dicte estrategias y políticas públicas para que los estudiantes puedan sacar provecho de la educación virtual y no se vean afectados (Calics \& Ochoa, 2021).

La investigación surge por la necesidad de conocer de qué manera las políticas públicas en la educación a distancia de la Institución Educativa 'Manuel Arévalo Cáceres- Los 
olivos", se relaciona con el logro de competencias en estudiantes de Educación Superior, mediante la investigación se plantea crear alternativas para que la situación académica 2021-1 no se vea afectada por la pandemia de la COVID-19, tales como implementar la plataforma MOODLE para el apoyo a los docentes en las tres carreras profesionales (Computación e Informática, Enfermería Técnica e industria Alimentaria), así como implementar actividades pedagógicas que se exige para las clases virtuales, ya sea en el uso de sílabos, archivos motivacionales, videos, audios, etc.

\section{Problema General}

- ¿De qué manera las Políticas Públicas en Educación a Distancia se relacionan con el Logro de Competencias en estudiantes de Educación Superior?

\section{Problemas específicos}

- ¿De qué manera la dimensión Organizativa se relaciona con en el Logro de Competencias de los estudiantes de Educación Superior?

- ¿De qué manera la dimensión Pedagógica se relaciona con el Logro de Competencias de los estudiantes de Educación Superior?

- ¿De qué manera la dimensión Tecnológica se relaciona con el Logro de Competencias de los estudiantes de Educación Superior?

\section{Objetivo General}

- Estudiar la incidencia de la Educación a Distancia como Política Pública en el Logro de Competencias en estudiantes de Educación Superior.

\section{Objetivos específicos}

- Determinar la incidencia de la dimensión Organizativa en el Logro de Competencias de los estudiantes de Educación Superior.

- Determinar la incidencia de la dimensión Pedagógica en el Logro de Competencias de los estudiantes de Educación Superior.

- Determinar la incidencia de la dimensión Pedagógica en el Logro de Competencias de los estudiantes de Educación Superior.

\section{Hipótesis General}

- Las Políticas Públicas en Educación a Distancia se relacionan con el Logro de Competencias en Estudiantes de Educación Superior.

\section{Hipótesis específicas}

- La dimensión Organizativa se relaciona con el Logro de Competencias en los 
estudiantes de Educación Superior.

- La dimensión Pedagógica se relaciona con el Logro de Competencias en los estudiantes de Educación Superior.

- La dimensión Tecnológica se relaciona con el Logro de Competencias en los estudiantes de Educación Superior.

\section{Antecedentes}

Hayashi et al. (2020) en su artículo 'Analysis of Public Policies in Distance Education in Brazil", tuvo como fin analizar las políticas públicas que se implementan en la educación de Brasil, de manera que se evidencie si su implementación y efectividad son las esperadas. Mediante una metodología bibliográfica exploratoria y explicativa, dando como conclusión que la modalidad virtual en las entidades públicas y privadas se encuentran operando de manera precaria y con dificultades, debido a que las políticas que se tienen no son contundentes, asimismo, las inversiones presupuestarias y la gestión que se tiene no son las convenientes ya que no se trata solo de adquirir recursos tecnológicos sino de innovar en herrmainetas y métodos que permitan a los estudiantes aprender.

De igual manera, Chambilla et al. (2021) en su tesis 'Project-based learning and its impact on the achievement of professional competencies in times of pandemic among students of the Professional School of Education of UNAMAD - 2020', en la universidad Nacional Amazónica de Madre de Dios. Esta investigación tuvo como objetivo determinar en que modo la aplicación del aprendizaje basado en proyectos se relaciona con el logro de competencias profesionales en tiempo de COVID-19, en base a una metodología mixta aplicada y explicativa, con un diseño experimental y la técnica de la observación. Se concluyó que, una correcta aplicación de ABP genera impactos positivos en la vida estudiantil, asimismo, contribuye a la gestión académica, de modo que desarrollan habilidades necesarias para desenvolver en el mercado laboral.

Godoy (2016) en su investigación 'Public policies for the assessment of quality of the Brazilian higher education system', del Centro Universitario Autónomo de Brasil, para la cual se obtuvo como objetivo principal estudiar la relación entre las políticas públicas en base a la educación de calidad y el carácter de bien público. Esta investigación se realizó mediante una metodología deductiva doctrinal, para la cual se obtuvo como resultado que la institución debe plantear el uso de políticas que resuelvan dos funciones, mejorar el desarrollo de los estudiantes y reflexionar sobre su identidad institucional, 
asimismo, hizo énfasis en que la cultura de Brasil no se prima un proyecto de autoevaluación.

Bautista et al. (2021) en su investigación "Achievement of competencies in the learning process during times of COVID-19', en el cual se tuvo como objetivo analizar el factor que limita el logro de las competencias en la educación básica, para tal propósito se utilizó un enfoque cuantitativo de nivel descriptivo y con diseño no experimental, con una muestra de 212 docentes. Se concluyó que por la falta de un portafolio con evidencia y una retroalimentación existen limitaciones en el aprendizaje, asimismo, el uso de tecnologías y la necesidad de conectividad no llegan a articular los saberes y por tal motivo se planteó como solución fortalecer las competencias de los docentes y mejorar los dos puntos antes mencionados, de manera que los estudiantes puedan desenvolver adecuadamente y aumentar sus conocimientos.

De Souza et al. (2018) en el artículo de investigación " Políticas Públicas em Educação Superior a Distância - Um estudo sobre a experiência do Consórcio Cederj', presentó como objetivo general dar a conocer el panorama de las políticas públicas en la educación virtual, esto con el fin de estudiar si se respeta la legislación y regulación en el Consorcio Cecierj. Entre sus consideraciones finales se evidenció que la virtualidad que se tiene se basa en avances tecnológicos y argumentos que permiten contribuir el crecimiento económico y la inclusión que se encuentra más alejada, asimismo, el sistema dispone de materiales de estudio y herramientas que permiten que los estudiantes puedan aprovechar esta disciplina, cabe señalar que la virtualidad no se da al 100\%, la entidad también imparte ciertos cursos semipresenciales.

\section{Teorías}

La educación virtual posee tres teorías: Teoría de la autonomía, Teoría de la interacción y la Teoría de la industrialización. En primer lugar, Carmona (2019) cree que, en la Teoría de la interacción, en la educación virtual las personas son autoresponsables de como aprenden, ya que este tipo de educación facilita la independencia, los alumnos deben tomar sus propias decisiones en base al alcance de objetivos y metas. En segundo lugar, Jiménez (2017) precisa que educación presencial se caracteriza por una estrecha relación comunicacional e interacción, sin embargo, en la educación virtual la comunicación es restringida, pese a que otorga mas dinamismo. Finalmente, se plantea que la educación a distancia es producto de la era industrial, en base al avance tecnológico, el cual permite 
que la enseñanza pueda ser masiva pero que depende de la planeación, además, cabe señalar que en esta teoría cada persone tiene una función dentro del conjunto, del mismo modo, plantea que se trata de esparcir crecimiento a largo plazo, más no ofrecer eficiencia.

\section{ESTRATEGIAS METODOLÓGICAS O MATERIALES Y MÉTODOS}

\section{Tipo y Diseño de Investigación}

La presente investigación tuvo como objetivo general, estudiar la incidencia de la Educación a Distancia como Política Pública en el Logro de Competencias en estudiantes de Educación Superior. Se tuvo una investigación cuantitativa básica y de exploración pura, teórica e indiscutible, es decir se recopilará información para las bases teóricas de las respectivas variables, de tal manera que se ahonde en el tema. Aplicada y de nivel correlacional, ya que se intenta medir la relación entre ambas variables, sin que el investigador interfiera o controle alguna de ellas, de tal modo que en este estudio se busca establecer y evidenciar la relación entre las variables de estudio. Se utilizó el diseño de investigación no experimental transeccional descriptiva, debido a que no se buscó cambiar el contexto, sino estudiarlas en él.

\section{Población y Muestra}

- Como población se consideró a 115 estudiantes del último año de estudios del Área Académica de Computación e Informática y docentes, del IESTP. "Manuel Arévalo Cáceres" - Los Olivos, Lima, Perú.

- Como muestra se seleccionó 80 estudiantes del último año de estudios del Área Académica de Computación e Informática y 10 docentes, del IESTP. "Manuel Arévalo Cáceres" - Los Olivos, Lima, Perú, es decir, un total de 90 personas

\section{Técnica e Instrumento de Recolección de Datos}

La técnica utilizada para llevar a cabo la investigación, es la encuesta en escala de Likert y como instrumento de recolección de datos el cuestionario, uno para cada variable, el cual será aplicado a la muestra en estudio.

\section{Procedimientos}

La recolección de información se realiza en tres pasos, el primer paso se centra en obtener datos importantes acerca del centro en investigación y de los sujetos de estudio, asimismo, se obtuvo un permiso de datos e información general, de modo que estos datos sean confidenciales. El segundo paso se centra en el contraste de fuentes en base a información recolectada de otras investigaciones, nacionales e internacionales, esto para que el 
presente estudio pueda tener información variada y verídica. El tercer paso se centra en la realización de un cuestionario acerca de las variables, de tal manera que la muestra en estudio pueda resolver las dudas y brindar información de primera mano, es importante señalar que fue validado debidamente por los expertos en base a la prueba Binominal, seguidamente se analizará en una base de datos, mediante uso de tablas y gráficos.

\section{Método de Análisis de Datos}

Asimismo, se utilizó la escala de Likert y el formulario Google forma para que la información sea ordenada y precisa, y como instrumento de recolección el cuestionario. Del mismo modo, para las respuestas de los instrumentos se usó como tabulación el programa estadístico SPSS V26, con la ayuda del método estadístico descriptivo: El cual es un método que permite que la investigación pueda obtener tablas y gráficos con los datos recogidos. Por último, se hizo uso del método estadístico inferencial, esto con el fin de contrastar las hipótesis de investigación planteadas en base al Alfa de Cronbach.

\section{RESULTADOS Y DISCUSIÓN}

\section{A. Resultados}

A continuación, se describen los resultados mediante tablas que contienen datos recopilados a partir de las encuestas aplicadas y realizadas a la muestra de investigación, estas se encuentran procesadas de acuerdo a las variables.

\section{Correlación de Rho Spearman entre las variables de estudio:}

Tabla 1 Correlación entre variables Políticas Públicas en Educación a Distancia y el Logro de Competencias de Educación Superior.

\begin{tabular}{|c|c|c|c|c|}
\hline \multicolumn{5}{|c|}{ Correlaciones } \\
\hline & & & $\begin{array}{l}\text { Políticas } \\
\text { Públicas en } \\
\text { Educación a } \\
\text { Distancia } \\
\end{array}$ & $\begin{array}{c}\text { Logro de } \\
\text { Competencias }\end{array}$ \\
\hline \multirow[t]{2}{*}{ Rho de Spearman } & $\begin{array}{l}\text { Politicas Públicas en } \\
\text { Educación a Distancia }\end{array}$ & $\begin{array}{l}\text { Coeficiente de correlación } \\
\text { Sig. (bilateral) } \\
\text { N }\end{array}$ & $\begin{array}{r}1,000 \\
90 \\
\end{array}$ & $\begin{array}{r}.413^{-} \\
.000 \\
90 \\
\end{array}$ \\
\hline & Logro de Competenciss & $\begin{array}{l}\text { Coeficiente de correlación } \\
\text { Sig. (bilateral) } \\
\text { N }\end{array}$ & $\begin{array}{r}.413^{-} \\
.000 \\
90 \\
\end{array}$ & $\begin{array}{r}1,000 \\
90 \\
\end{array}$ \\
\hline
\end{tabular}

Fuente: Elaboración propia.

Interpretación: Se aprecia en la tabla 1 que existe un impacto significativo positivo entre las variables "políticas públicas en educación a distancia' y el ' 'logro de competencias", mediante la elaboración de la correlación de Spearman, la cual obtuvo una cifra de p (sig) menor que $0.05(0,000<0.05)$ y un $\mathrm{r}=0,413$, cuyo valor es débil y de nivel medio, pero 
permite validar la relación entre ambas variables planteadas por el investigador.

\section{Correlación del Objetivo General}

HG: Las Políticas Públicas en Educación a Distancia se relacionan con el Logro de Competencias en Estudiantes de Educación Superior.

Ho: Las Políticas Públicas en Educación a Distancia NO se relacionan con el Logro de Competencias en Estudiantes de Educación Superior.

Tabla 2 Prueba de Hipótesis General

\begin{tabular}{|c|c|c|c|c|c|}
\hline & Valor & $\mathrm{gl}$ & $\begin{array}{c}\text { Sig. asintótica (2 } \\
\text { caras) }\end{array}$ & $\begin{array}{c}\text { Significación } \\
\text { exacta ( } 2 \text { caras) }\end{array}$ & $\begin{array}{l}\text { Significación } \\
\text { exacta (1 cara) }\end{array}$ \\
\hline Chi-cuadrado de Pearson & $15,368^{*}$ & 1 & .000 & \multirow{6}{*}{.000} & \multirow{6}{*}{.000} \\
\hline Corrección de continuidad & 13,625 & 1 & .000 & & \\
\hline Razón de verosimilitud & 15,750 & 1 & .000 & & \\
\hline Prueba exacta de Fisher & & & & & \\
\hline Asociación linesl por linesl & 15,198 & 1 & .000 & & \\
\hline $\mathrm{N}$ de casos válidos & 90 & & & & \\
\hline
\end{tabular}

Fuente: Elaboración propia.

Interpretación: Se aprecia en la Tabla 2 que, con un $X^{2}=15,369, \mathrm{gl}=1$ y p=0.000; el valor de $\mathrm{p}$ (sig) fue menor que $0.05(0,000<0.05)$, entonces se rechaza la hipótesis nula $\mathrm{H}_{0}$ y se acepta la hipótesis planteada por el investigador $\mathrm{H}_{\mathrm{G}}$ : 'Las Políticas Públicas en Educación a Distancia se relacionan con el Logro de Competencias en Estudiantes de Educación Superior'”.

\section{Correlación del Objetivo Específico 1}

H1: La dimensión Organizativa se relaciona con el Logro de Competencias en los estudiantes de Educación Superior.

Ho: La dimensión Organizativa NO se relaciona con el Logro de Competencias en los estudiantes de Educación Superior.

Tabla 3 Prueba de Chi- Cuadrado de la Hipótesis específica 1

\begin{tabular}{|c|c|c|c|c|c|}
\hline & Valor & $\mathrm{gl}$ & $\begin{array}{c}\text { Sig. asintótica (2 } \\
\text { caras) }\end{array}$ & $\begin{array}{c}\text { Significación } \\
\text { exacta }(2 \text { caras) }\end{array}$ & $\begin{array}{l}\text { Significación } \\
\text { exacta (1 cara) }\end{array}$ \\
\hline Chi-cuadrado de Pearson & $9,723^{\mathrm{x}}$ & 1 & .002 & & \\
\hline Corrección de continuidad" & 8,443 & 1 & .004 & & \\
\hline Razón de verosimilitud & 8,884 & 1 & .002 & & \\
\hline Prueba exacta de Fisher & & & & .003 & .002 \\
\hline Asociación lineal por lineal & 9,615 & 1 & .002 & & \\
\hline N de casos válidos & 90 & & & & \\
\hline
\end{tabular}

Fuente: Elaboración propia. 
Interpretación: Se aprecia en la Tabla 3 que, con un $X^{2}=9,723, g l=1$ y p=0.000; el valor de $\mathrm{p}$ (sig) fue menor que $0.05(0,002<0.05)$, entonces se rechaza la hipótesis nula $\mathrm{H}_{0}$ y se acepta la hipótesis planteada por el investigador $\mathrm{H}_{1}$ : ' $\mathrm{La}$ dimensión Organizativa se relaciona con el Logro de Competencias en los estudiantes de Educación Superior.',

\section{Correlación del Objetivo Específico 2}

H2: La dimensión Pedagógica se relaciona con el Logro de Competencias en los estudiantes de Educación Superior.

Ho: La dimensión Pedagógica NO se relaciona con el Logro de Competencias en los estudiantes de Educación Superior.

Tabla 4 Prueba de Chi- Cuadrado de la Hipótesis específica 2

\begin{tabular}{|c|c|c|c|c|c|}
\hline & Valor & gl & $\begin{array}{c}\text { Sig. asintótica (2 } \\
\text { caras) }\end{array}$ & $\begin{array}{c}\text { Significación } \\
\text { exacta ( } 2 \text { caras) }\end{array}$ & $\begin{array}{c}\text { Significación } \\
\text { exacta (1 cara) }\end{array}$ \\
\hline Chi-cuadrado de Pearson & $13,166^{*}$ & 1 & .000 & & \\
\hline Corrección de continuidad ${ }^{\prime}$ & 11,379 & 1 & .001 & & \\
\hline Razón de verosimilitud & 13,629 & 1 & .000 & & \\
\hline Prueba exacta de Fisher & & & & .001 &, 000 \\
\hline Asociación lineal por linesl & 13,019 & 1 & .000 & & \\
\hline N de casos válidos & 90 & & & & \\
\hline
\end{tabular}

Fuente: Elaboración propia.

Interpretación: Se aprecia en la Tabla 4 que, con un $X^{2}=13,166, \mathrm{gl}=1$ y p=0.000; el valor de $\mathrm{p}$ (sig) fue menor que $0.05(0,000<0.05)$, entonces se rechaza la hipótesis nula $\mathrm{H}_{0}$ y se acepta la hipótesis planteada por el investigador $\mathrm{H}_{2}$ : ' $L a$ dimensión Pedagógica se relaciona con el Logro de Competencias en los estudiantes de Educación Superior’'.

\section{Correlación del Objetivo Específico 3}

H3: La dimensión Tecnológica se relaciona con el Logro de Competencias en los estudiantes de Educación Superior.

Ho: La dimensión Tecnológica NO se relaciona con el Logro de Competencias en los estudiantes de Educación Superior.

Tabla 5 Prueba de Chi-Cuadrado de la Hipótesis específica 3

\begin{tabular}{|l|r|r|rr|}
\hline & \multicolumn{1}{|c|}{ Valor } & \multicolumn{1}{|c|}{ gl } & Sig. asintótica (2 cargs) &, 000 \\
\hline Chi-cuadrado de Pearson & $30,037^{*}$ & 2 & &, 000 \\
Razón de verosimilitud & 33,555 & 2 &, 000 \\
Ascciación lineal por linesl & 27,830 & 1 & \\
N de casos válidos & 90 & & &, \\
\hline
\end{tabular}

Fuente: Elaboración propia. 
Interpretación: Con un $\mathrm{X}^{2}=30,037, \mathrm{gl}=2$ y p=0.000; el valor de $\mathrm{p}$ (sig) fue menor que $0.05(0,000<0.05)$, entonces se rechaza la hipótesis nula $\mathrm{H}_{0}$ y se acepta la hipótesis planteada por el investigador $\mathrm{H}_{3}$ : ' $L a$ dimensión Tecnológica se relaciona con el Logro de Competencias en los estudiantes de Educación Superior'”.

\section{B. Discusión}

En este punto se presenta la discusión de dichos resultados encontrados a partir de los objetivos planteados, con apoyo de los antecedentes y teorías encontradas, describiendo los fenómenos para dar respuesta a la problemática definida.

Las Políticas Públicas en Educación a Distancia se relacionan con el Logro de Competencias en Estudiantes de Educación Superior, ya que como se refleja en el estudio de Paucca et al. (2021), en el cual se analizó el logro de competencias en el sector educativo, dando como resultado que es un fenómeno que permite mejorar la toma de decisiones y alcanzar los objetivos planteados, mediante un Rho de Spearman r=.718 y $\mathrm{p}=.000<0.05$, confirmando la hipótesis del investigador. Asimismo, se concluyó que el logro de competencias en la educación es sumamente importante, ya que los alumnos aprenden y los docentes otorgan una retroalimentación mediante el uso de la tecnología, potenciando la enseñanza y el aprendizaje. Por tal motivo, las instituciones educativas ahora deben apoyarse en la tecnología para dictar conocimientos y habilidades, en las cuales el docente será un factor clave.

Del mismo modo, la dimensión Organizativa se relaciona con el Logro de Competencias en los estudiantes de Educación Superior, teniendo resultados positivos al igual que Chambilla et al. (2021) en su tesis ', dando como resultado que el logro de competencias profesionales también se da de manera adecuada en la educación a distancia, pese a los desafíos actuales que se tienen, esto debido a que los recursos tecnológicos ofrecen una dimensión organizativa para el ordenamiento de las áreas y estrategias, en función del compromiso con la enseñanza. Además, el lenguaje utilizando redes sociales u otras plataformas de enseñanza resultan ser un foco ameno para el aprendizaje, ya que otorga dinamismo y ofrece un impacto significativo en el desarrollo crítico y creativo.

La dimensión Pedagógica se relaciona con el Logro de Competencias en los estudiantes de Educación Superior, lo cual posee un parecido con la investigación de Hayashi (2020) en ya que los procesos comunicativos en la educación presencial se han visto afectados con su paso a la virtualidad, y por tal motivo resulta importante que los elementos y 
herramientas que se tengan sean adecuados, asimismo, debe haber un espectro que sirva para orientar y motivar a los estudiantes, ya sea organizando actividades grupales y gestionar los materiales para que ellos puedan sentirse cómodos y aprender eficazmente, y no sea un retroceso en la enseñanza. Es por esto que las políticas públicas que incluyan métodos pedagógicos ayudarán a superar los desafíos e integrar a los individuos implicados.

Además, la existencia de una relación entre la dimensión Tecnológica y el logro de competencias en los estudiantes de Educación Superior, se vincula con la investigación de Bautista et al. (2021) ya que el uso de la tecnología si no se emplea adecuadamente no se estaría llegando a la articulación de los saberes y competencias, en esta modalidad las sesiones de enseñanza necesitan una conectividad y emplear una tecnología capaz para que los estudiantes puedan tener una retroalimentación y evaluación pertinente. Para tal propósito, los alumnos deben tener una orientación continua, es decir los docentes poseen gran responsabilidad en la formación de sus estudiantes, pero también en el uso de las TICS, dependiendo se su compromiso y actitud para construir conocimiento, ya que las tecnologías pueden resultar beneficiosa, sin embargo, también puede ocasionar problemas y una desconexión en la enseñanza.

\section{CONCLUSIÓN O CONSIDERACIONES FINALES}

\section{Conclusiones}

- Se logró evidenciar que existe una relación significativa entre las políticas públicas y el logro de competencias en estudiantes de Educación Superior. Mediante la elaboración del Chi-Cuadrado de Pearson, se obtuvo la cifra de 15.369, cuyo valor es de cualidad fuerte y alta, por lo tanto, la hipótesis general planteada por el investigador es aceptada.

- Se logró evidenciar la existencia de una relación entre la dimensión organizativa y el logro de competencias en los estudiantes de Educación Superior. Mediante la elaboración del Chi- Cuadrado de Pearson, se obtuvo la cifra de 9.723, siendo un valor de nivel medio, por lo tanto, la hipótesis específica 1 planteada por el investigador es validada y aceptada.

- Se logró evidenciar la relación entre la dimensión pedagógica y el logro de competencias en los estudiantes de Educación Superior. Mediante la elaboración del 
Chi-Cuadrado de Pearson, se obtuvo una cifra de 13.166, cuyo valor alto es fuerte y moderado, por lo tanto, se acepta la hipótesis específica 2.

- Se logró evidenciar la existencia de una relación entre la dimensión Tecnológica y el logro de competencias en los estudiantes de Educación Superior. Mediante la elaboración del Chi- Cuadrado de Pearson, obteniendo una cifra de 30.037, cuyo valor es fuerte y sólido, permitiendo la aceptación de la hipótesis específica 3.

\section{Recomendaciones}

- Se recomienda que la institución de Educación Superior, en vista del cambio a la virtualización de sus clases, sigan empleando el uso de las plataformas como Moodle y Classroom, ya que se evidencia que ambas son recursos didácticos que favorecen y motivan a los estudiantes, asimismo, permiten una comunicación directa con el docente.

- Se recomienda a la institución Educativa Superior que brinde capacitaciones constantes en gestión de plataformas virtuales, seguimiento online, formación docente, entre otras herramientas que permitan que el factor humano pueda permitir que los estudiantes aprovechen al máximo la virtualidad.

- Se recomienda a la institución Educativa Superior que favorezca el desarrollo de la dimensión pedagógica en la educación virtual, mediante estrategias que permitan facilitar la formación y el aprendizaje en los estudiantes, así como brindar un acompañamiento que esté orientado a generar cambios positivos en los actores involucrados.

- Se recomienda a la institución Educativa Superior que el aprendizaje virtual en los estudiantes sea dinámico e interactivo, asimismo, que se fomenten planes que permitan profundizar en los beneficios de las tecnologías y su formación estudiantil, de modo que los alumnos puedan.

\section{LISTA DE REFERENCIAS}

Ankney, J. (2017). A virtual Learning System's impact on Student Achievement in a Secondary Biology College Preparatory course, an Action Research study. Degree of Doctor , University of South Carolina. Obtenido de https://scholarcommons.sc.edu/cgi/viewcontent.cgi?article=5288\&context=etd

Avalos, K., \& Brenes, P. (2021). The impact of covid-19 in higher education classes, in the english teaching for I and II cycle mayor at Universidad Hispanoamericana. 
Cárdenas Díaz...

Ciencia Latina Revista Científica Multidisciplinar, 5(5). doi:https://doi.org/10.37811/cl_rcm.v5i5.919

Bautista, T., Santa María, H., \& Córdova, U. (2021). Achievement of competencies in the learning process during times of COVID-19. Propósitos y Representaciones, 9(1), 1-15. doi:http://dx.doi.org/10.20511/pyr2021.v9n1.1175

Boboc, M., \& Koc, S. (2019). Student-Centered virtual Learning Environments in Higher Education. (J. Keengwe, Ed.) United States of America : IGI Global.

Calics , L., \& Ochoa, L. (2021). Educational public policies and quality in basic primary education: an analysis from theoretical foundations. Ciencia Latina Revista $\begin{array}{lll}\text { Científica } \quad \text { Multidisciplinar, } & \text { 5127-5138. }\end{array}$ doi:https://doi.org/10.37811/cl_rcm.v5i4.680

Carmona, D. (2019). Autonomy and Interdependence. $\square$ e Ethics of Care and Disability. Revista Humanity, 10(2), 3-18. doi:: https://doi.org/10.15517/h.v10i2.41154

Chambilla, Y., Puma, M., \& Bejar, C. (2021). Project-based learning and its impact on the achievement of professional competencies in times of pandemic among students of the Professional School of Education of UNAMAD - 2020. Universidad Nacional Amazónica de Madre de Dios Puerto Maldonado. doi:https://doi.org/10.37811/cl_rcm.v5i3.505

Chiappe, A., \& Wills, A. (2021). Crowd based Open Online Education as an alternative to he Covid-19 educationarl crisis. Ensaio, 1-20. Obtenido de https://www.scielo.br/j/ensaio/a/mrpS9cWgBzgQbZsmVQMkvWv/?format=pdf \&lang=en

Dauzón, L., \& Izquierdo, M. (2020). Digital natives and technology for L2 learning outside of the classroom. Apertura, 12(1), 72-87. doi:https://doi.org/10.32870/ap.vl2nl.1801

De Souza, G., Carvalho, A., \& Chrispino, Á. (2018). Políticas Públicas em Educação Superior a Distância - Um estudo sobre a experiência do Consórcio Cederj. Ensaio: Avaliação e Políticas Públicas em Educação, 26(99), 445-470. doi:https://doi.org/10.1590/S0104-403620180026000938

Educación, M. d. (2020). Diario El Peruano. Resolución Viceministerial N ${ }^{\circ}$ 084-2020MINEDU. Obtenido de 
https://cdn.www.gob.pe/uploads/document/file/574783/RVM_N_084-2020MINEDU.pdf

Godoy, A. (2016). Public policies for the assessment of quality of the Brazilian higher education system. Revista de Investigacoes, 3(3), 53-69. doi:doi.org/10.5380/rinc.v3i3.49033

Gómez, I., \& Escobar, F. (2021). Virtual education in times of pandemic: Increasing social inequality in Peru. Educación y Ciudad, 171. doi:https://doi.org/10.1590/SciELOPreprints.1996

Hayashi, C., Dos Santos, F., \& Rodrígues, F. (2020). Analysis of Public Policies in Distance Education in Brazi. Researh, Society and Development, 9(1), 1-18. doi:http://dx.doi.org/10.33448/rsd-v9i1.1667

Herrera, L. (2017). Impact of implementing a virtual learning environment (VLE) int the

EFL Classroom. Íkala, Revist Cultura, 22(3), 479-498. doi:https://doi.org/10.17533/udea.ikala.v22n03a07

Higuera, A., \& Rivera, E. (2021). Academic Performance in Virtual Learning Environments During Covid-19 Pandemic in Higher Education. Scielo Preprint, 1-24. doi:https://doi.org/10.1590/SciELOPreprints.2862

Jiménez, J. (2017). A theory of sociality as interaction: Towards a naturalistic, universal and interactional social analysis. Cinta Moebio, 157-171. doi:10.4067/S0717$554 X 2017000200157$

Karasel, N., Bastas, M., Altinay, F., Altinay, Z., \& Dagli, G. (2020). Distance Education for Students with Special Needs in Primary Schools in the Period of CoVid-19. 8(3). doi:https://dx.doi.org/10.20511/pyr2020.v8n3.587

Naveros, W. (2020). Adopción del uso de las TIC como gestión de políticas públicas educativas en Institución Educativa 2 de Mayo, Calleo 2019. Tesis de maestría, Universidad César Vallejo. Obtenido de https://repositorio.ucv.edu.pe/bitstream/handle/20.500.12692/44704/Naveros_R WA-SD.pdf?sequence $=1 \&$ is Allowed $=\mathrm{y}$

Paucca, N., Alfaro, M., García, J., Ramírez, J., \& Rafayle, R. (2021). Investigación formativa y logro de competencias en estudiantes de una universidad pública Lima. PURIQ, 3(2), 365-384. doi:https://doi.org/10.37073/puriq.3.2.167 
Perú, P. d. (2020). Decreto de Urgencia $\mathrm{N}^{\circ}$ 026-2020. Obtenido de https://cdn.www.gob.pe/uploads/document/file/566447/DU026-202018649481.pdf

Pires, C., Zen, P., Freitas, F., Arruda, M., \& Gemperli, R. (2020). Online learning and COVID-19: a meta-synthesis analysis. CLinics, 1-4. doi:https://doi.org/10.6061/clinics/2020/e2286

Sierralta, S. (2021). Competencias digitales en tiempos de COVID-19, reto para los maestros de la Institución Educativa CECAT "Marcial Acharán". MendiveRevista de educación, 19(3), 755-763. Obtenido de http://scielo.sld.cu/scielo.php?script=sci_arttext\&pid=S1815$76962021000300755 \&$ lang=es

Sood, N. (2016). Distance Education. United States: Vikas. 